\title{
Listes des opérations, si déjà, alors correctement!
}

\author{
Jürg Unger-Köppela, Josef Emil Brandenberg ${ }^{b}$, Urs Stoffel ${ }^{c}$ \\ ${ }^{a}$ Dr méd., membre du Comité central de la FMH, responsable du département Médecine et tarifs hospitaliers; ${ }^{b}$ Dr méd., président de I'Association Suisse des \\ médecins avec activité chirurgicale et invasive, $\mathrm{fmCh} ;{ }^{\circ} \mathrm{Dr}$ méd., membre du Comité central de la FMH, responsable du département Médecine et tarifs ambulatoires
}

Les premières discussions entre l'OFSP et la FMH sur le transfert des interventions hospitalières vers le secteur ambulatoire ont eu lieu à l'automne 2016. Sur le fond, la FMH soutient le transfert d'interventions hospitalières se prêtant à un traitement ambulatoire, mais en même temps elle exige une rémunération appropriée.

Le département Médecine et tarifs hospitaliers a pris ce dossier en main en concertation avec ses collègues des tarifs ambulatoires et de l'organisation faîtière la plus concernée par la question, la fmCh. Parallèlement, nous avons constaté qu'entre toutes les idées de solutions contradictoires mises sur la table pour faire évoluer le système de santé de notre pays, il n'était guère possible de frapper un grand coup pour régler tous les problèmes. Il est beaucoup plus prometteur d'acquérir des expériences au travers de projets pilotes clairs et délimités, et d'incorporer ensuite ces expériences dans des solutions plus globales. Après la publication, par Lucerne et Zurich, de listes des opérations qui doivent être impérativement réalisées en ambulatoire, la FMH a contacté la direction de la santé de ces cantons. Nous leur avons proposé de développer ensemble un projet pilote dans lequel la tarification et le financement des opérations en question seraient réglés de manière nouvelle, et évalués simultanément dans le

L'indication selon laquelle une opération est réalisée avec ou sans hospitalisation relèvera toujours exclusivement de la décision du médecin.

cadre d'une étude concomitante. Notre proposition a rencontré un écho positif et les discussions avec les différents protagonistes sont en cours.

Pour la FMH et la fmCh, le point le plus important de ce projet est le suivant: l'indication selon laquelle une opération est réalisée avec ou sans hospitalisation a toujours relevé et continuera de relever exclusivement de la décision du médecin. Tous les partenaires soutiennent ce principe. En plus des indications liées à la maladie, des impératifs psycho-sociaux peuvent aussi rendre une hospitalisation nécessaire pour une intervention. Le contrôle permettant de déterminer si l'indication d'une hospitalisation a été posée correctement doit être réduit à une démarche administrative minimale. Divers modèles avec des contrôles effectués par le canton ou la caisse-maladie avant l'intervention et d'autres avec une vérification ultérieure de l'indication sont actuellement en discussion.

Il est indispensable que TOUTES les listes contiennent les mêmes opérations et que les processus de vérification soient partout aussi simples, afin de garantir la sécurité juridique et de limiter l'administration au minimum. Les structures qui fonctionnent doivent être utilisées pour éviter de créer des postes supplémentaires auprès des caisses, des cantons ou des fournisseurs de prestations, qui n'apporteraient aucun avantage direct aux patients. Dans ce sens, nous soutenons l'OFSP qui continue de se pencher sur la ques-

Les interventions des listes des opérations ont besoin d'un tarif identique pour une même prestation fournie en ambulatoire ou à l'hôpital.

tion en association avec les groupes impliqués dans l'optique d'une solution uniforme pour toute la Suisse. Nombre d'hôpitaux sont aujourd'hui axés principalement sur les opérations hospitalières et les interventions ambulatoires restent l'exception. C'est pourquoi les processus et les locaux hospitaliers doivent être adaptés afin de permettre une organisation ambulatoire efficace. C'est le seul moyen de créer une «ligne de production ambulatoire» pertinente, comme a déclaré le président de la fmCh. Les fournisseurs de prestations doivent par ailleurs disposer du temps nécessaire pour s'adapter. Les coûts d'une opération ambulatoire doivent être couverts. Avec le TARMED, version actuelle et future, ce n'est la plupart du temps pas le cas. Les interventions figurant sur les listes des opérations ont besoin d'un tarif identique pour une même prestation fournie en ambulatoire ou à l'hôpital. Une solution doit également être trouvée pour les patients assurés en privé.

Les projets pilotes constituent un bon moyen de mettre en œuvre et d'évaluer avec précision ces exigences primordiales sur le terrain. Les meilleurs résultats obtenus pourront ensuite être intégrés dans une législation uniforme pour toute la Suisse. 